\title{
FNDC5/irisin, a molecular target for boosting reward-related learning and motivation
}

\author{
Judit Zsuga $^{{ }^{*}}$, Gabor Tajti ${ }^{1}$, Csaba Papp $^{1}$, Bela Juhasz ${ }^{2}$, Rudolf Gesztelyi ${ }^{3}$
}

${ }^{1}$ Department of Health Systems Management and Quality Management for Health Care, Faculty of Public Health, University of Debrecen, Nagyerdei krt 98, 4032 Debrecen, Hungary

${ }^{2}$ Department of Pharmacology and Pharmacotherapy, Faculty of Medicine, University of Debrecen, Nagyerdei krt 98, 4032 Debrecen, Hungary

${ }^{3}$ Department of Pharmacology, Faculty of Pharmacy, University of Debrecen, Nagyerdei krt 98, 4032 Debrecen, Hungary

\section{Running title: 'FNDC5/irisin boosts reward-learning and motivation'}

* Corresponding author: Judit Zsuga; Cell: +36 30 625-0144; fax: +36 52 411-717 ext. 55187; e-mail: zsuga.judit@med.unideb.hu 


\begin{abstract}
Interventions focusing on the prevention and treatment of chronic non-communicable diseases are on rise. In the current article, we propose that dysfunction of the mesocortico-limbic reward system contributes to the emergence of the WHO-identified risk behaviors (tobacco use, unhealthy diet, physical inactivity and harmful use of alcohol), behaviors that underlie the evolution of major non-communicable diseases (e.g. cardiovascular diseases, cancer, diabetes and chronic respiratory diseases). Given that dopaminergic neurons of the mesocortico-limbic system are tightly associated with reward-related processes and motivation, their dysfunction may fundamentally influence behavior. While nicotine and alcohol alter dopamine neuron function by influencing some receptors, mesocortico-limbic system dysfunction was associated with elevation of metabolic set-point leading to hedonic over-eating. Although there is some empirical evidence, precise molecular mechanism for linking physical inactivity and mesocortico-limbic dysfunction per se seems to be missing; identification of which may contribute to higher success rates for interventions targeting lifestyle changes pertaining to physical activity.
\end{abstract}

In the current article, we compile evidence in support of a link between exercise and the mesocortico-limbic system by elucidating interactions on the axis of muscle - irisin - brain derived neurotrophic factor (BDNF) - and dopaminergic function of the midbrain. Irisin is a contraction-regulated myokine formed primarily in skeletal muscle but also in the brain. Irisin stirred considerable interest, when its ability to induce browning of white adipose tissue parallel to increasing thermogenesis was discovered. Furthermore, it may also play a role in the regulation of behavior given it readily enters the central nervous system, where it induces BDNF expression in several brain areas linked to reward processing, e.g. the ventral tegmental area and the hippocampus. BDNF is a neurotropic factor that increases neuronal dopamine content, modulates dopamine release relevant for neuronal plasticity and increased neuronal survival as well as learning and memory. Further linking BDNF to dopaminergic function is BDNF's ability to activate tropomyosin-related kinase B receptor that shares signalization with presynaptic dopamine-3 receptors in the ventral tegmental area.

Summarizing, we propose that the skeletal muscle derived irisin may be the link between physical activity and reward-related processes and motivation. Moreover alteration of this axis may contribute to sedentary lifestyle and subsequent non-communicable diseases. Preclinical and clinical experimental models to test this hypothesis are also proposed. 


\section{General background}

Physical inactivity, unhealthy diet, tobacco use and harmful use of alcohol are identified by the World Health Organization (WHO) as key risk factors for major chronic non-communicable diseases (NCDs) primarily responsible for premature death worldwide, e.g. cardiovascular diseases, cancer, diabetes and chronic inflammatory lung disease $(1,2)$.

It is clear that combating NCDs is one of the greatest pressing challenges high-income countries must face, a challenge articulated in and reflected by several international policies, such as the Global Action Plan for the Prevention and Control of NCDs 2013-2020 resolution (1). This global action plan posits a paradigm shift by providing a road map and a menu of policy options that if implemented collectively could halt the rise in diabetes and obesity, lead to a $25 \%$ relative reduction in risk of premature mortality from cardiovascular diseases, cancer, diabetes, and chronic respiratory diseases.

Furthermore, a relative reduction in the prevalence of risk factors such as insufficient physical activity and tobacco use in persons aged 15+ years, and harmful use of alcohol, is anticipated by $10 \%, 30 \%$ and $10 \%$, respectively (1). Nonetheless rather than risk factors, all of them could be viewed as risk behaviors, with lifestyle changes or behavioral modifications being amongst the most effective means for preventing or slowing the progression of NCDs.

In our current work, we put forward the hypothesis that the common denominator of risk behaviors underlying the most burdening NCDs may be the dysfunction (or untoward function) of the mesocortico-limbic system. Furthermore we propose that the irisin - brain derived neurotrophic factor (BDNF) pathway forms a significant the link between physical inactivity and the mesocortico-limbic system.

A common denominator for change of behavior is motivation and allied reward-related processes driving change of said behavior. Motivation and reward processing are tightly linked to mesocortico-limbic dopaminergic activation, as laid out by the two major theoretical frameworks, the reward-prediction error and the incentive salience hypothesis (3-5).

According to the reward prediction error hypothesis phasic dopamine response signals the discrepancy between expected and actual reward value of a cue governing future goal-directed activity $(5,6)$. Placing this concept into the reinforcement learning paradigm, the reward prediction error signal emitted by dopamine neurons is the neural correlate of model-free reinforcement learning's prediction error and is used to compute the value of state without any attempt to build a model. This model-free system interacts with the model-based system (7) that 
uses a model, possibly utilizing the continuous function of the brain's default network, incorporating structures such as the hippocampus and the orbitofrontal cortex (8).

On the other hand, Berridge and colleagues posit that reward associated processes are conceptualized using three psychological components, e.g. liking (the hedonic value of a cue), reward learning by means of associative learning and incentive salience that is 'wanting' (motivational incentive of a cue) (9). Accordingly, it attributes incentive (motivational) value to a cue making it more (or alternatively less) 'wanted' (4). This theoretical framework offers a different interpretation of the role attributed to mesocortico-limbic dopaminergic activation as it pointed to dopamine's causal involvement in incentive salience as opposed to prior beliefs linking dopamine to hedonic attribute of cues $(4,10)$. Summarizing, incentive salience is the Pavlovian-guided attribution of motivational value to a previously reward-related neutral representation of a cue (conditioned stimulus (CS)) that results in a more attractive and 'wanted' cue/stimulus. Accordingly, the incentive salience value of a cue is the net of associativelearning derived prior knowledge concerning the relationship between the cue and the reward (unconditioned stimulus (UCS)) (11).

Either way, the central role of the dopaminergic mesocortico-limbic system has to be acknowledged (Fig. 1).

Substantial evidence may be retrieved tying the influence of alcohol and nicotine to the mesocortico-limbic reward structures in the contemporary literature of addiction (for review see $(12,13))$. There are also elaborate reviews focusing on the 'hedonic control of eating' (1416), moreover recently the term 'hedonic obesity' was coined to describe a form of obesity, in which the metabolic set-point is elevated due to hedonic over-eating linked to mesocorticolimbic system dysfunction (17). Nonetheless, there seems to be missing a link between physical inactivity and mesocortico-limbic dysfunction per se, identification of which may contribute to higher success rates for interventions targeting lifestyle changes pertaining to physical activity. In the following section we will compile evidence in support of the link between exercise and the mesocortico-limbic system by elucidating interactions on the axis of skeletal muscle - irisin - BDNF - and dopaminergic function of the midbrain (Fig. 2). 


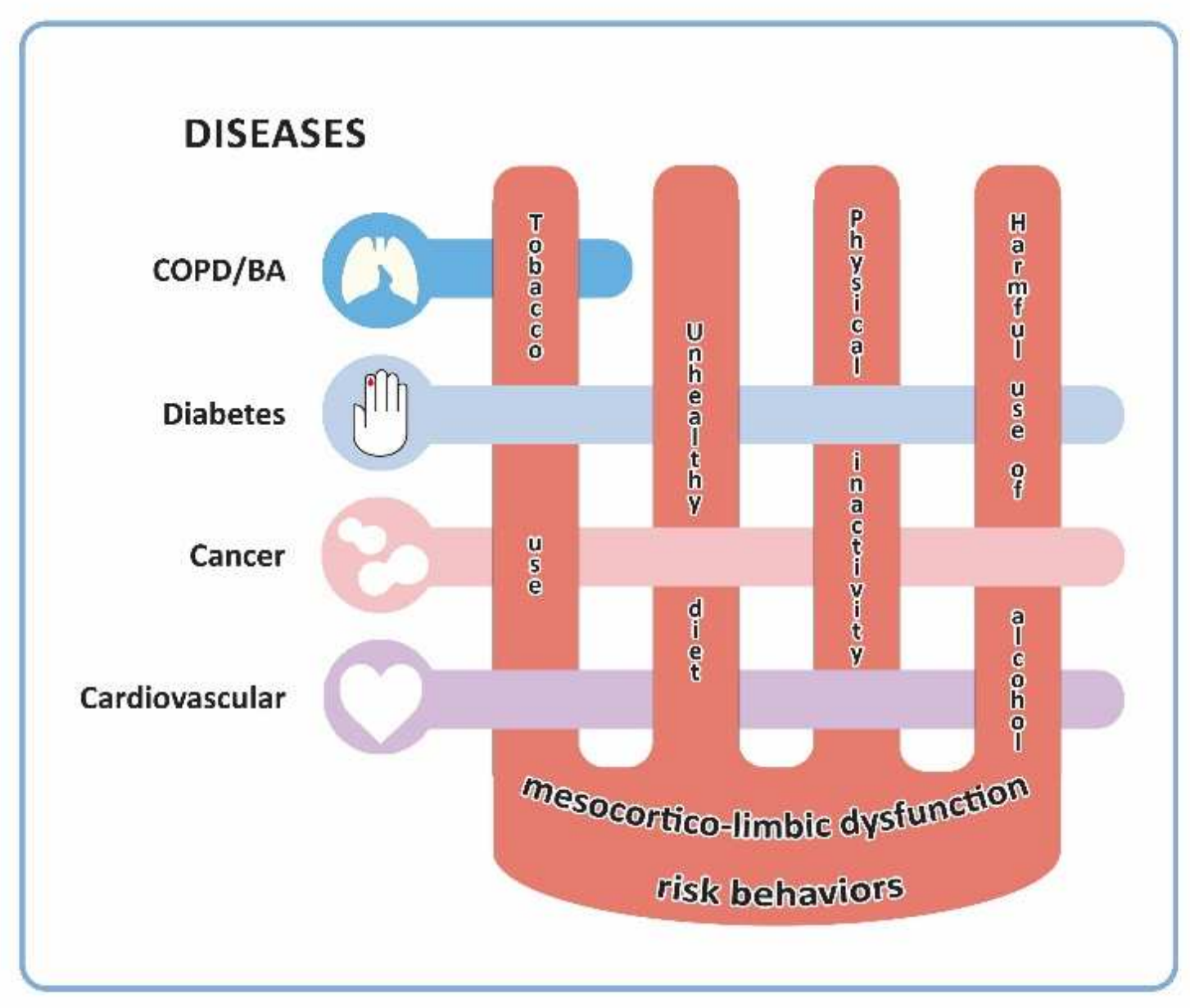

Figure 1. Dysfunction (or untoward function) of the mesocortico-limbic system may be a causative factor in development of risk behaviors leading to the evolution of major NCDs. Risk behaviors and NCDs are indicated as conceptualized by the WHO for the purpose of Global Action Plan for the Prevention and Control of NCDs 2013-2020 (1). Abbreviations: COPD: chronic obstructive pulmonary disease, BA: bronchial asthma 


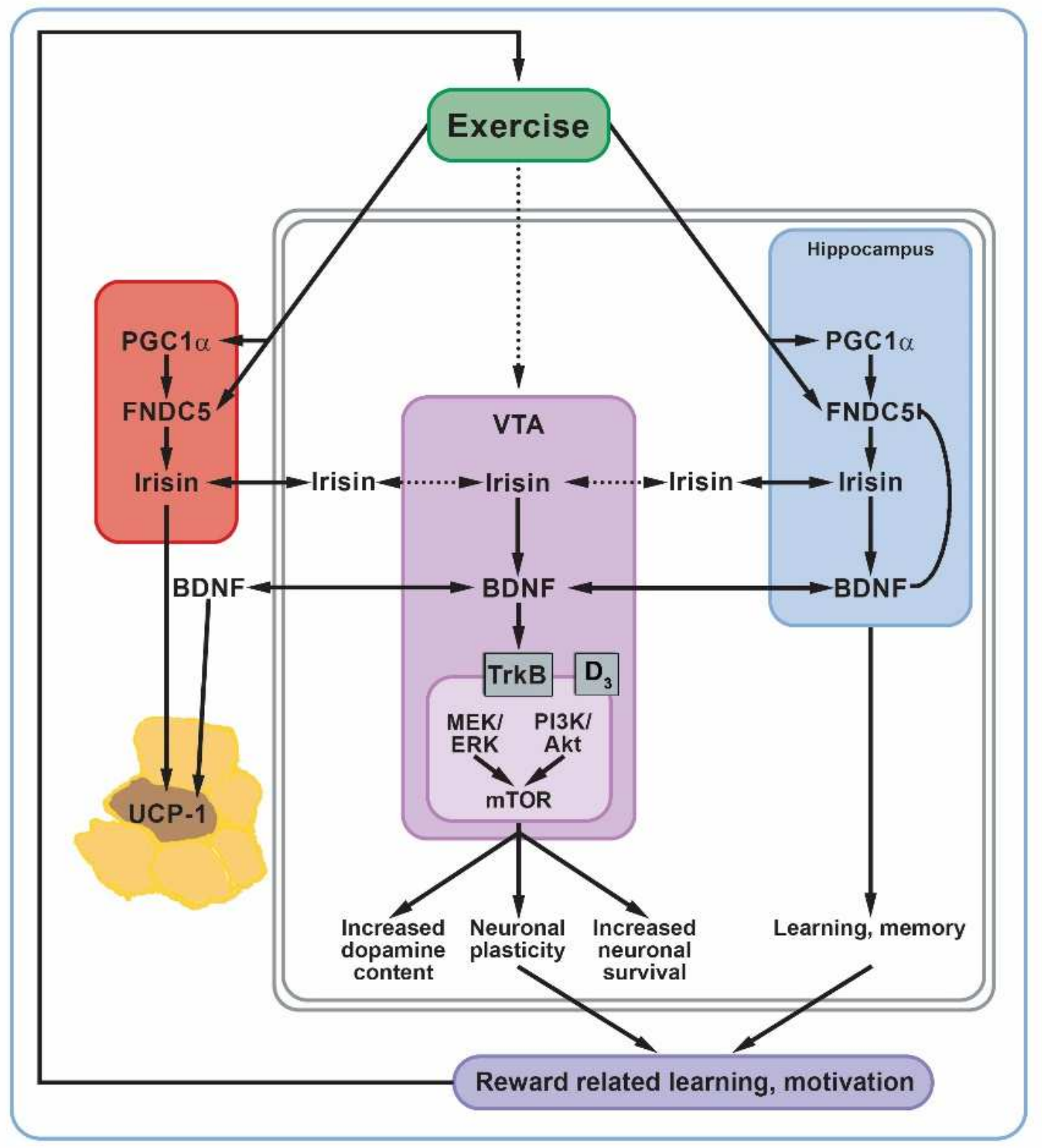

Figure 2. The effect of exercise on irisin-BDNF pathway and related neuronal and metabolic changes. Physical activity increases irisin levels by inducing the expression of both FNDC5 (parent molecule of irisin) and its upstream regulator PGC1 $\alpha$ and FNDC5. After proteolytic cleavage of FNDC5, irisin is released mainly from skeletal muscle (red box) but also from several brain areas (e.g.: hippocampus). Circulating irisin, on one hand, induces expression of UCP-1 in adipose tissue that induces thermogenesis by browning of beige adipocytes. On the other hand it irisin upregulates BDNF expression in many brain areas (VTA, hippocampus, etc.). Both irisin and BDNF readily cross the blood-brain-barrier thus irisin formed in the periphery is able to increase BDNF levels in the brain, and BDNF formed in the brain may influence peripheral tissues such as adipocytes. We propose that exercise induced increase in irisin levels leads to elevated BDNF in the brain that, by activating TrkB receptors located on dopaminergic neurons in the VTA, may exert considerable influence on the dopamine content, neuronal survival and plasticity leading to altered reward-related learning and motivation, 
processes that may underlie the evolution of behaviors. There are considerable interactions between these molecular pathways as the ability of BDNF to inhibit FNDC5 expression was shown previously (18). Furthermore, BDNF released from the CNS was shown to induce UPC1 expression and browning in peripheral adipocytes (19). It is worthy of notice that the signalization of TrkB and $\mathrm{D}_{3}$ dopamine receptors overlap. Dotted lines indicate connections suggested by the hypothesis outlaid in the current article. Abbreviations: Akt: protein kinase B, BDNF: brain-derived neurotrophic factor, CNS: central nervous system, $\mathrm{D}_{3}$ : dopamine 3 receptor, ERK: extracellular signal-related kinase, FNDC5: fibronectin type III domaincontaining 5, MEK: mitogen-activated protein kinase kinase, mTOR: mammalian target of rapamycin, PGC1 $\alpha$ : peroxisome proliferator-activated receptor-gamma coactivator $1 \alpha, \mathrm{PI} 3 \mathrm{~K}$ : phosphoinositide 3-kinase, UCP-1: uncoupling protein 1, TrkB: tropomyosin-related kinase B, VTA: ventral tegmental area

\section{Emergence of skeletal muscle as an endocrine organ}

The identification of several muscle-derived cytokines and peptides termed myokines has led to the re-conceptualization of skeletal muscle as an endocrine organ $(20,21)$. Myokines, by definition, are produced, expressed, and released by muscle fibers and exert local or remote effects in an autocrine/paracrine or endocrine fashion, respectively. Myokines may be clustered based on their postulated function e.g. their contribution to metabolism, angiogenesis and myogenesis (22). One subset of myokines, contraction-regulated myokines, has raised considerable interest recently, based on its potential to account for the beneficial effects of exercise, given that it allegedly assumes a fundamental role for the interplay between skeletal muscle, adipose tissue and brain (21).

One aspect of muscle-fat-brain crosstalk relevant for exercise-related beneficial effects of myokines concerns the browning of white adipose tissue (23). Conventionally, white adipose tissue (WAT) and brown adipose tissue (BAT) are differentiated based on their developmental origin and the role they assume in energy homeostasis. While WAT serves as the primary energy storing organ enabling prolonged survival in the absence of meals, BAT is responsible for energy dissipation in the form of non-shivering thermogenesis. In line with this, expression of mitochondrial uncoupling protein UCP1, a key protein underlying thermogenic activity of BAT, is low in WAT and high in BAT (24). Nonetheless, recently an intermediate form of adipocytes was identified called beige or brite (brown in white) adipocytes $(25,26)$. These cells originate from cell lines resembling that of WAT and are consistently found at anatomical locations typical for WAT. At baseline, they have low expression of UCP1. Nevertheless, if activated these beige adipocytes have the capability to switch from energy storing to energy 
dissipating mode, by changing from a baseline expression to increased expression of UCP1, parallel to phenotypic alterations rendering these activated beige cells phenotypically similar to BAT. In fact, it is now proposed that in humans BAT may be mostly made-up of beige adipocytes (27). It is expected that the identification of molecular determinants for browning will yield novel therapeutic targets for combating some risk factors of major NCDs (23). To date, of the several factors identified as relevant for browning of beige adipocytes, irisin, a contraction-regulated myokine, has stirred considerable interest based on its exercise related beneficial effects $(28,29)$.

\section{A putative contraction-regulated myokine: irisin}

Irisin was first identified by Boström and colleagues in mice and humans (30). It is a highly conservative $12 \mathrm{kDa}$ polypeptide showing 100\% homology of its amino acid sequence among most mammals, indicating a highly preserved function (31). Irisin is formed by proteolytic cleavage of the transmembrane protein fibronectin type III domain containing 5 (FNDC5). Expression of FNDC5 is regulated by peroxisome proliferator-activated receptor-gamma coactivator protein-1 $\alpha(\mathrm{PGC} 1 \alpha)$, a transcriptional co-activator also known for regulating oxidative metabolism in BAT (32). Both the expression of PGC1 $\alpha$ and FNDC5 show positive correlation with physical activity as well as with each other, reflected by their increased or decreased expression due to sustained physical training or sedentary lifestyle, respectively (33, 34). Following the proteolytic cleavage of FNDC5, irisin is released into the circulation. Albeit FNDC5/irisin expression is most abundant in skeletal muscle, other tissues such as the adipose tissue (rendering irisin an adipokine as well) $(31,35)$, tongue, rectum, and brain also express FNDC5 and irisin. In addition, the kidney, liver and lung also contain irisin in low levels (36).

The most profound effect of irisin is the activation of oxygen consumption and thermogenesis of fat cells (32) by induction of WAT browning in selected regions (30). A recent report showed the beneficial effect of recombinant irisin in mice by decreasing body weight and improving glucose homeostasis parallel to upregulating the expression of thermogenic genes such as UCP1 and PGC1 $\alpha$, possibly via p38 mitogen-activated protein kinase (p38 MAPK) and extracellular signal-related kinase (ERK) pathways (37). Furthermore using Laser-scanning cytometry, Kristof and colleagues were able to demonstrate irisin's efficacy to induce beige differentiation of subcutaneous white adipocytes in human samples (38). Based on its influence on adipose tissue, the possible contribution of irisin to obesity and related metabolic disorders, such as 
insulin resistance, Type II diabetes and polycystic ovarium syndrome has been proposed (29, $36)$, although relating data are inconsistent at present $(29,36)$.

While circulating irisin establishes a firm link between skeletal muscle and adipose tissue, it also exerts influence in the central nervous system (CNS). In rodents, FNDC5 mRNA was isolated in several structures such as the midbrain and the hippocampus (32), structures known for being involved in reward-related model-free and model-based reinforcement learning (8). Conversely, others have provided strong evidence for endurance exercise to elevate neuronal FNDC5 expression in the hippocampus of mice (18). In addition, exercise-derived irisin produced in the periphery is able to cross the blood-brain barrier (32). A further indirect evidence supporting the link between exercise and alteration of PGC $1 \alpha /$ FNDC5 pathway comes from a study on mice where markers of brain and muscle mitochondrial biogenesis (including PGC1 $\alpha$ ) were quantified following 8 weeks of endurance training using a treadmill run to fatigue paradigm. The authors showed a significantly increased PGC1 $\alpha$ level in muscle and several brain regions, including those involved in reward-related processes, e.g. the frontal lobe, hippocampus and midbrain, when compared with sedentary counterparts (39). Furthermore, the finding that the level of FNDC5 expression is reduced in PGC1 $\alpha-/-$ mice indicates the ability of PGC1 $\alpha$ to induce neuronal FNDC5 expression, similarly to that in muscle (18). Based on these results, the possible induction of FNDC5 expression in these areas may be postulated.

A significant effect of neuronal FNDC5/irisin is the ability to induce BDNF expression in several brain areas. For example, forced hippocampal expression of FNDC5 induced hippocampal expression of BDNF per se and elevation of the level of irisin in the circulation also increased BDNF expression in the hippocampus. Furthermore, manipulations of FNDC5 expression by means of RNAi, small noncoding RNA transcripts that regulate mRNA expression-mediated knockdown in cortical neurons, showed corresponding decrease of cortical BDNF expression as well (18).

\section{BDNF, a link between FNDC5/irisin and mesocortico-limbic system}

BDNF is a neurotrophin assumed to possess significant role in the maturation, preservation and plasticity of the brain (40) as BDNF modulates the release of neurotransmitters, especially dopamine and serotonin (41). Conversely, lower BDNF levels influence the strength of synaptic connection (42), evoke long-term potentiation inherent of reward-learning related changes, consequently resulting in modified behavior (43). Additionally, BDNF has recently emerged as 
a possible mediator of cognition-enhancing effects of exercise and intermittent fasting (44). Voluntary aerobe exercise enhances cognitive performance and increases serum BDNF levels in both humans and rodents (45-47). The exercise-responsive upregulation and subsequent release of FNDC5/irisin from the periphery together with the direct upregulation of FNDC5 in the neurons jointly contribute to the upregulation of BDNF (18).

BDNF is synthetized throughout the neurons of the CNS including the midbrain's ventral tegmental area, hippocampus and peripheral organs such as skeletal muscle, liver and adipose tissue (44). Thus, BDNF distribution shows a pattern similar to that of FNDC5. BDNF is synthetized in a pre-pro form and released into the circulation either as mature BDNF (this being the active form and is referred to as BDNF further on) or pro-BDNF (converted to BDNF in the plasma). BDNF readily crosses the blood-brain barrier and exerts its effects by binding to its high affinity receptor tropomyosin-related kinase B (TrkB), a tyrosine kinase receptor (44).

Several lines of evidence underline the connection between BDNF and the mesocortico-limbic system, with BDNF being expressed (43) and affording direct influence on brain circuits activated during reward-processing (48). BDNF plays a role in the development of dopaminergic system, as it seems to be a potent trophic factor in rat and human mesencephalic dopaminergic neuron cultures, reflected by increased survival and elevated neuronal dopamine and tyrosine hydroxylase content (43). Recently, microRNAs were identified in the midbrain dopaminergic neurons, microRNA124a specifically was shown to influence BDNF expression and related dopamine neuron survival and plasticity $(41,49)$.

Prior work has indicated that mesencephalic dopaminergic neurons express TrkB receptors rendering these neurons susceptible to autocrine and paracrine effects of BDNF (50). BDNFinduced TrkB receptor activation influences several signaling pathways including the phosphoinositide 3-kinase -Akt and MEK/ERK pathways (51) two signaling paths linked to dopamine $3\left(\mathrm{D}_{3}\right)$ receptor signalization, as well (52). In fact, presynaptic $\mathrm{D}_{3}$ receptor expression depends on BDNF levels in reward-related dopaminergic neurons of the ventral tegmental area $(53,54)$. Upon their stimulation, $\mathrm{D}_{3}$ receptors mediate dopamine's effect on structural plasticity via phosphorylation of MEK/ERK and PI3/Akt/mTOR pathways, paths also being under the control of BDNF (52). Specific activation of $\mathrm{D}_{3}$ receptors by quinpirole, a $\mathrm{D} 3$ receptor agonist, increased soma size and number as well as the length of primary dendrites, which effect may be inhibited by D3 receptor antagonists (52). 
Further robust evidence for BDNF's influence on the mesocortico-limbic system comes from substance abuse studies. It is well-established that the common neural structure underlying substance abuse is the mesocortico-limbic system $(52,55)$. Chronic drug use increases BDNF levels in the ventral tegmental area (VTA), concordantly BDNF infusion into this area induced behavioral effects characteristic of drug addiction including drug seeking and psychomotor agitation (56). In an elegant experiment, Vargas-Perez and colleagues showed that BDNF may be responsible for inducing a switch to a drug-dependent motivational state, reliant on the dopaminergic rewarding effects. They investigated BDNF's ability to change the neurobiological substrates mediating opioid reward and found that BDNF's ability to induce this transition is dependent on dopamine, as this effect could be readily blocked by administration of the dopamine receptor antagonist alpha-flupenthixol (57). Likewise, in humans the single nucleotide polymorphism of BDNF resulting in val66met substitution influenced the activity of neurotransmitters involved in reward processes alongside with alterations in neural responses to reward and alcohol-related risk phenotypes (48).

Summarizing the above-mentioned findings, we propose that the exercise-related myokine irisin forms a possible link between sedentary lifestyle and mesocortico-limbic processes such as reward learning and motivation by influencing BDNF's effect in reward-related structures, e.g. VTA and hippocampus. Given the findings in clinical investigations suggestive of the presence of irisin resistance in the periphery (31), similar mechanism may be present on the muscle-brain axis contributing to the lack of motivation related to physical activity.

Based on prior works, our hypothesis is unique in a sense that it offers a novel, common pathomechanism at the heart of the evolution of NCDs that, upon using multidisciplinary approaches (involving genetic, behavioral and clinical data capture and analysis), could yield a novel framework for personalized therapeutic and preventive interventions.

\section{Models for investigation}

\section{Preclinical models}

The role that endurance training has on the FNDC5 expression and irisin level in reward-related structures may be assessed by experiments similar to that described by Steiner and colleagues, who investigated the influence of prolonged endurance training on neuronal changes of PGC1 $\alpha$ and other mitochondrial substrates in areas involved in reward processing (39). Given that only Boström's initial publication in 2012 (30) stirred considerable interest towards the role of 
increased FNDC5 expression and related irisin production, quantification of FNDC5 gene expression and irisin protein levels was omitted.

To elucidate the molecular mechanisms linking FNDC5 expression to elevated BDNF levels, in vitro investigations on human mesencephalic dopaminergic neuron cultures could be performed. Addition of upstream modulators of FNDC5 expression (e.g. PGC1 $\alpha$ ) or irisin per $s e$ to the culture medium followed by the mRNA and protein level quantification of molecules linked to already known (e.g. p38 MAPK or ERK) and putative signalization pathways of irisin along with BDNF levels could shed light on the possible mechanism.

\section{Interventional study on healthy male volunteers}

Given prior findings that circulating irisin levels are most reliably increased in energy deficient states (36), endurance exercise training for 10 weeks should be initiated in untrained male volunteers. Since skeletal muscle mass was found to be the main predictor of circulating irisin level, elevation of irisin should be normalized to either biceps circumference or fat-free mass. Following the establishment of increased circulating level of irisin, serum BDNF (a surrogate for BDNF level in the CNS, given its free passage through the blood-brain-barrier) should be assessed. To account for the alleged influence of altered BDNF levels have on reinforcement learning, the Iowa gambling task, developed to characterize decision-making in an experimental setting, could be used (58). Altered performance on the Iowa gambling task was shown to be linked to addiction, pathological gambling, obsessive-compulsive disorder and diseases that have a pathomechanism linked to dopamine neuron dysfunction of the midbrain $(59,60)$. In addition, change of functional connectivity of relevant structures (ventral striatum, hippocampus, orbitofrontal cortex, amygdala) may be assessed by task fMRI (60). Furthermore, combined PET-fMRI examinations by enabling the conjoint acquisition of molecular, functional and anatomical information may shed light to the relevance of the physical activityirisin-BDNF-dopamine axis. Using PET tracers (e.g. $\left[{ }^{11} \mathrm{C}\right]$ raclopride and $\left[{ }^{18} \mathrm{~F}\right]$ fallypride specific for dopamine $\mathrm{D}_{2} / \mathrm{D}_{3}$ receptors, 6- $\left[{ }^{18} \mathrm{~F}\right]$ fluoro-L-DOPA for characterizing presynaptic dopamine level and turnover or $\left[{ }^{11} \mathrm{C}\right] \mathrm{McN} 5652$ for assessing dopamine transporter binding (61)) dopaminergic function in the VTA may be characterized, while simultaneously assessing neural activity and metabolism, information derived from fMRI's BOLD signal. These, together with changes in functional connectivity of reward-related regions, could yield new means for understanding of behavioral changes (e.g. endurance exercise) reward and motivation in health and disease states. 


\section{Acknowledgement}

This study was supported by the Hungarian Brain Research Program (KTIA_13_NAP-A-V/2) and TÁMOP 4.2.2.A-11/1/KONV-2012-0023 project. The TÁMOP project is co-financed by the European Union and the European Social Fund.

\section{Conflict of interest statement}

None of the authors have any conflict of interest.

\section{References}

1. World Health Organization. Global action plan for the prevention and control of noncommunicable diseases 2013-2020. . 2013.

2. Mitchell NS, Catenacci VA, Wyatt HR, Hill JO. Obesity: overview of an epidemic. Psychiatr Clin North Am. 2011;34(4):717-32.

3. Colombo M. Deep and beautiful. the reward prediction error hypothesis of dopamine. Studies in History and Philosophy of Science Part C: Studies in History and Philosophy of Biological and Biomedical Sciences. 2014;45:57-67.

4. Berridge KC. The debate over dopamine's role in reward: the case for incentive salience. Psychopharmacology (Berl). 2007 Apr;191(3):391-431.

5. Schultz W, Dayan P, Montague PR. A neural substrate of prediction and reward. Science. 1997 Mar 14;275(5306):1593-9.

6. Schultz W. Behavioral dopamine signals. Trends Neurosci. 2007 May;30(5):203-10.

7. Daw ND, Gershman SJ, Seymour B, Dayan P, Dolan RJ. Model-based influences on humans' choices and striatal prediction errors. Neuron. 2011 Mar 24;69(6):1204-15.

8. Zsuga J, Biro K, Papp C, Tajti G, Gesztelyi R. The "proactive" model of learning: Integrative framework for model-free and model-based reinforcement learning utilizing the associative learning-based proactive brain concept. Behav Neurosci. 2016;130(1):6.

9. Berridge KC. From prediction error to incentive salience: mesolimbic computation of reward motivation. Eur J Neurosci. 2012 Apr;35(7):1124-43.

10. Berridge KC, Robinson TE. What is the role of dopamine in reward: hedonic impact, reward learning, or incentive salience? Brain Res Rev. 1998;28(3):309-69. 
11. Zhang J, Berridge KC, Tindell AJ, Smith KS, Aldridge JW. A neural computational model of incentive salience. PLoS Comput Biol. 2009;5(7):e1000437.

12. Nutt DJ, Lingford-Hughes A, Erritzoe D, Stokes PR. The dopamine theory of addiction: 40 years of highs and lows. Nature Reviews Neuroscience. 2015;16(5):305-12.

13. Söderpalm B, Ericson M. Neurocircuitry involved in the development of alcohol addiction: the dopamine system and its access points. In: Behavioral neurobiology of alcohol addiction. Springer; 2013. p. 127-61.

14. Saper CB, Chou TC, Elmquist JK. The need to feed: homeostatic and hedonic control of eating. Neuron. 2002;36(2):199-211.

15. Berthoud $\mathrm{H}$. The neurobiology of food intake in an obesogenic environment. Proc Nutr Soc. 2012;71(04):478-87.

16. Seeley RJ, Berridge KC. The Hunger Games. Cell. 2015;160(5):805-6.

17. Yu Y, Vasselli J, Zhang Y, Mechanick J, Korner J, Peterli R. Metabolic vs. hedonic obesity: a conceptual distinction and its clinical implications. Obesity Reviews. 2015.

18. Wrann CD, White JP, Salogiannnis J, Laznik-Bogoslavski D, Wu J, Ma D, et al. Exercise Induces Hippocampal BDNF through a PGC-1a/FNDC5 Pathway. Cell Metabolism. 2013;18:1-11.

19. Cao L, Choi EY, Liu X, Martin A, Wang C, Xu X, et al. White to brown fat phenotypic switch induced by genetic and environmental activation of a hypothalamic-adipocyte axis. Cell metabolism. 2011;14(3):324-38.

20. Pedersen BK, Akerstrom TC, Nielsen AR, Fischer CP. Role of myokines in exercise and metabolism. J Appl Physiol (1985). 2007 Sep;103(3):1093-8.

21. Pedersen BK, Febbraio MA. Muscles, exercise and obesity: skeletal muscle as a secretory organ. Nature Reviews Endocrinology. 2012;8(8):457-65.

22. Yoon JH, Kim J, Song P, Lee TG, Suh P, Ryu SH. Secretomics for skeletal muscle cells: a discovery of novel regulators? Advances in biological regulation. 2012;52(2):340-50.

23. Contreras C, Gonzalez F, Fernø J, Diéguez C, Rahmouni K, Nogueiras R, et al. The brain and brown fat. Ann Med. 2015;47(2):150-68.

24. Cannon B, Nedergaard J. Brown adipose tissue: function and physiological significance. Physiol Rev. 2004 Jan;84(1):277-359.

25. Seale P, Bjork B, Yang W, Kajimura S, Chin S, Kuang S, et al. PRDM16 controls a brown fat/skeletal muscle switch. Nature. 2008;454(7207):961-7.

26. Ishibashi J, Seale P. Medicine. Beige can be slimming. Science. 2010 May 28;328(5982):1113-4. 
27. Wu J, Boström P, Sparks LM, Ye L, Choi JH, Giang A, et al. Beige adipocytes are a distinct type of thermogenic fat cell in mouse and human. Cell. 2012;150(2):366-76.

28. Lee P, Linderman JD, Smith S, Brychta RJ, Wang J, Idelson C, et al. Irisin and FGF21 are cold-induced endocrine activators of brown fat function in humans. Cell metabolism. 2014;19(2):302-9.

29. Chen J, Huang Y, Gusdon AM, Qu S. Irisin: a new molecular marker and target in metabolic disorder. Lipids in health and disease. 2015;14(1):1-6.

30. Boström P, Wu J, Jedrychowski MP, Korde A, Ye L, Lo JC, et al. A PGC1-[agr]-dependent myokine that drives brown-fat-like development of white fat and thermogenesis. Nature. 2012;481(7382):463-8.

31. Roca-Rivada A, Castelao C, Senin LL, Landrove MO, Baltar J, Crujeiras AB, et al. FNDC5/irisin is not only a myokine but also an adipokine. PloS one. 2013;8(4):e60563.

32. Phillips C, Baktir MA, Srivatsan M, Salehi A. Neuroprotective effects of physical activity on the brain: a closer look at trophic factor signaling. Frontiers in cellular neuroscience. 2014;8.

33. Handschin C, Spiegelman BM. The role of exercise and PGC1 1 in inflammation and chronic disease. Nature. 2008;454(7203):463-9.

34. Lecker SH, Zavin A, Cao P, Arena R, Allsup K, Daniels KM, et al. Expression of the irisin precursor FNDC5 in skeletal muscle correlates with aerobic exercise performance in patients with heart failure. Circ Heart Fail. 2012 Nov;5(6):812-8.

35. Novelle MG, Contreras C, Romero-Picó A, López M, Diéguez C. Irisin, two years later. International journal of endocrinology. 2013;2013.

36. Huh JY, Panagiotou G, Mougios V, Brinkoetter M, Vamvini MT, Schneider BE, et al. FNDC5 and irisin in humans: I. Predictors of circulating concentrations in serum and plasma and II. mRNA expression and circulating concentrations in response to weight loss and exercise. Metab Clin Exp. 2012;61(12):1725-38.

37. Zhang Y, Li R, Meng Y, Li S, Donelan W, Zhao Y, et al. Irisin stimulates browning of white adipocytes through mitogen-activated protein kinase p38 MAP kinase and ERK MAP kinase signaling. Diabetes. 2014 Feb;63(2):514-25.

38. Kristóf E, Doan-Xuan Q, Bai P, Bacso Z, Fésüs L. Laser-scanning cytometry can quantify human adipocyte browning and proves effectiveness of irisin. Scientific reports. 2015;5.

39. Steiner JL, Murphy EA, McClellan JL, Carmichael MD, Davis JM. Exercise training increases mitochondrial biogenesis in the brain. J Appl Physiol (1985). 2011 Oct;111(4):106671.

40. Chao Mx, Rajagopal R, Lee Fx. Neurotrophin signalling in health and disease. Clin Sci. 2006;110:167-73. 
41. Bahi A, Dreyer J. Striatal modulation of BDNF expression using microRNA124aexpressing lentiviral vectors impairs ethanol-induced conditioned-place preference and voluntary alcohol consumption. Eur J Neurosci. 2013;38(2):2328-37.

42. Huang T, Lee C, Liu Y. Serum brain-derived neurotrophic factor levels in patients with major depression: effects of antidepressants. J Psychiatr Res. 2008;42(7):521-5.

43. Yan Q, Feng M, Yan S. Different expression of brain-derived neurotrophic factor in the nucleus accumbens of alcohol-preferring (P) and-nonpreferring (NP) rats. Brain Res. 2005;1035(2):215-8.

44. Marosi K, Mattson MP. BDNF mediates adaptive brain and body responses to energetic challenges. Trends in Endocrinology \& Metabolism. 2014;25(2):89-98.

45. Griffin EW, Mullally S, Foley C, Warmington SA, O'Mara SM, Kelly AM. Aerobic exercise improves hippocampal function and increases BDNF in the serum of young adult males. Physiol Behav. 2011;104(5):934-41.

46. Vaynman S, Ying Z, Gomez-Pinilla F. Hippocampal BDNF mediates the efficacy of exercise on synaptic plasticity and cognition. Eur J Neurosci. 2004;20(10):2580-90.

47. Kobilo T, Liu QR, Gandhi K, Mughal M, Shaham Y, van Praag H. Running is the neurogenic and neurotrophic stimulus in environmental enrichment. Learn Mem. 2011 Aug 30;18(9):605-9.

48. Nees F, Witt S, Dinu-Biringer R, Lourdusamy A, Tzschoppe J, Vollstädt-Klein S, et al. BDNF Val66Met and reward-related brain function in adolescents: Role for early alcohol consumption. Alcohol. 2015;49(2):103-10.

49. Chandrasekar V, Dreyer J. microRNAs miR-124, let-7d and miR-181a regulate cocaineinduced plasticity. Molecular and Cellular Neuroscience. 2009;42(4):350-62.

50. Numan S, Seroogy KB. Expression of trkB and trkC mRNAs by adult midbrain dopamine neurons: A double-label in situ hybridization study. J Comp Neurol. 1999;403(3):295-308.

51. Chen MJ, Russo-Neustadt AA. Exercise activates the phosphatidylinositol 3-kinase pathway. Mol Brain Res. 2005;135(1):181-93.

52. Collo G, Cavalleri L, Spano P. Structural plasticity in mesencephalic dopaminergic neurons produced by drugs of abuse: critical role of BDNF and dopamine. Frontiers in pharmacology. $2014 ; 5$.

53. Guillin O, Griffon N, Bezard E, Leriche L, Diaz J, Gross C, et al. Brain-derived neurotrophic factor controls dopamine D3 receptor expression: therapeutic implications in Parkinson's disease. Eur J Pharmacol. 2003;480(1):89-95.

54. Jeanblanc J, He DY, McGough NN, Logrip ML, Phamluong K, Janak PH, et al. The dopamine D3 receptor is part of a homeostatic pathway regulating ethanol consumption. $\mathbf{J}$ Neurosci. 2006 Feb 1;26(5):1457-64. 
55. Covey DP, Roitman MF, Garris PA. Illicit dopamine transients: reconciling actions of abused drugs. Trends Neurosci. 2014;37(4):200-10.

56. Bolaños CA, Nestler EJ. Neurotrophic mechanisms in drug addiction. Neuromolecular medicine. 2004;5(1):69-83.

57. Vargas-Perez H, Ting-A Kee R, Walton CH, Hansen DM, Razavi R, Clarke L, et al. Ventral tegmental area BDNF induces an opiate-dependent-like reward state in naive rats. Science. 2009 Jun 26;324(5935):1732-4.

58. Bechara A, Damasio AR, Damasio H, Anderson SW. Insensitivity to future consequences following damage to human prefrontal cortex. Cognition. 1994;50(1):7-15.

59. Brevers D, Bechara A, Cleeremans A, Noël X. Iowa Gambling Task (IGT): twenty years after-gambling disorder and IGT. Frontiers in psychology. 2013;4.

60. Li X, Lu Z, D'Argembeau A, Ng M, Bechara A. The Iowa gambling task in fMRI images. Hum Brain Mapp. 2010;31(3):410-23.

61. Mier W, Mier D. Advantages in functional imaging of the brain. Frontiers in Human Neuroscience. 2015;9:249. 